International Journal of Medical Sciences

(c) Ivyspring International Publisher. All rights reserved

Case report

\title{
A case of mistaken identity: Asystole causing convulsions identified using implantable loop recorder.
}

\author{
Khalil Kanjwal, Beverly Karabin, Yousuf Kanjwal, Blair P Grubb ${ }^{\bowtie}$
}

Electrophysiology Section, Division of Cardiology. Department of Medicine, The University of Toledo Medical Center, Health Science Campus, Toledo OH, USA

Corresponding author: Blair P Grubb, MD, Director Electrophysiology Services, Division of Cardiology, Department of Medicine, Health Sciences Campus, University of Toledo Medical Center, Mail Stop 1118, 3000 Arlington Ave., Toledo OH 43614. USA. Phone 419-3833778; Fax: 419-383-3041.

Received: 2010.06.10; Accepted: 2010.06.20; Published: 2010.06.21

\begin{abstract}
We present herein an interesting tracing of a patient who suffered from recurrent episodes of transient loss of consciousness (TLOC) associated with convulsive activity thought to be due to epilepsy or conversion disorder.
\end{abstract}

Key words: Asystole, implantable loop recorder, transient loss of consciousness

\section{Case description}

A thirty four year old woman was referred to our syncope and autonomic disorder center for evaluation of recurrent unexplained periods of transient loss of consciousness (TLOC) associated with convulsive activity. The episodes would come on suddenly while sitting or standing with little or no prodrome. She would abruptly lose consciousness and fall to the floor. Bystanders reported her to be pale and ashen in color. Witnesses reported that during episodes she would display tonic-colonic like convulsive activity lasting from 1-5 minutes associated with urinary incontinence. The loss of consciousness could last for 30 to 45 minutes. Afterwards the patient was confused and fatigued for the remainder of the day. The patient had undergone multiple evaluations including, 12 lead electrocardiograms, echocardiography, stress testing, tilt table testing and prolonged holter and event monitoring, all of which were unremarkable. Repeated electroencephalograms (including a prolonged inpatient monitoring) were inconclusive and empiric therapy with multiple anti- seizure medications did not alter the frequency or severity of her TLOC. She was labeled as having either refractory epilepsy or conversion disorder. After presentation to our center she underwent placement of an implantable loop recorder (ILR). She later suffered one of her typical TLOC episode associated with witnessed convulsive activity. A download of the device demonstrated that concomitant with the episode of TLOC the ILR had recorded a periods of complete heart block followed by a prolonged periods of asystole, with artifacts consistent with convulsive activity was noted. Prolonged periods of asystole have been reported to result in convulsive activity that may be misdiagnosed as being due to epilepsy $(1,2,3)$. The patient then underwent permanent pacemaker implantation with complete resolution of her TLOC episodes.

This case graphically illustrates the utility of the ILR in establishing the cause of recurrent unexplained TLOC. In addition the tracing demonstrates an interesting sequence of complete heart block followed by prolonged asystole that resulted in TLOC with convulsive activity. Prolonged asystole has been reported to cause abrupt onset, convulsive episodes with prolonged loss of consciousness that at times can be mis- 
diagnosed as epilepsy $(1,2,3)$. An ILR can be invaluable in identifying these patients and facilitating prompt therapy (3).

\section{Conflict of Interest}

The authors have declared that no conflict of interest exists.

\section{References}

1. Zaidi A, Clough P, Cooper P, Scheepers B, Fitzpatrick AP. Misdiagnosis of epilepsy: many seizure-like attacks have a cardiovascular cause. J Am Coll Cardiol. 2000;36(1):181-4

2. Kanjwal K, Kanjwal Y, Beverly K, Grubb BP. Clinical Symptoms Associated with asystolic or bradycardic responses on implantable loop recorder monitoring in patients with recurrent syncope. Int J Med Sci 2009; 6:106-110.

3. Kanjwal K, Karabin B, Kanjwal Y, Grubb BP. Differentiation of Convulsive syncope from Epilepsy with an Implantable Loop Recorder. Int J Med Sci 2009; 6(6):296-300.

\section{Figures}
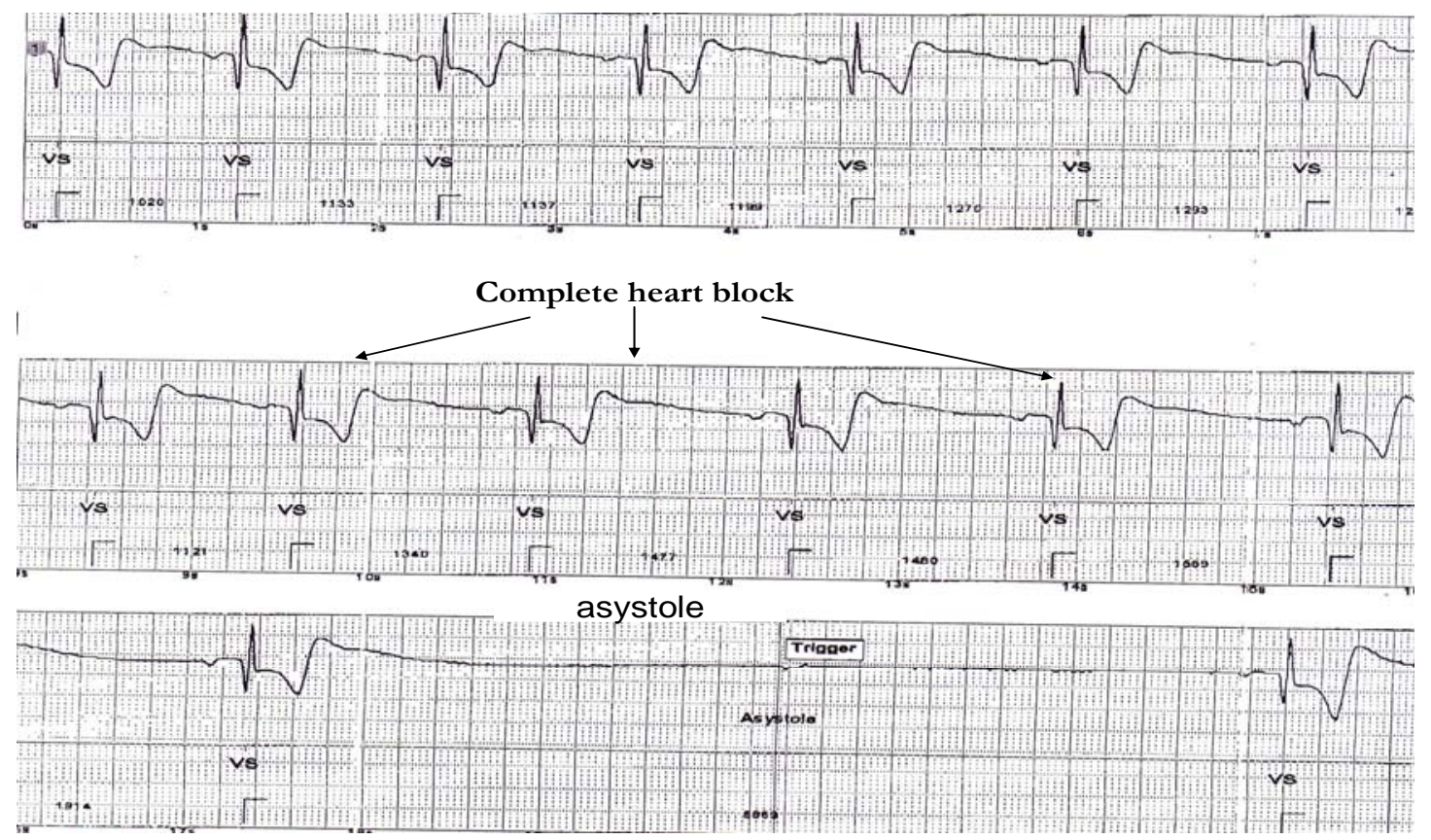

Figure I: Tracings downloaded from implantable loop recorder shows transition from sinus rhythm to complete heart block and prolonged asystole. 

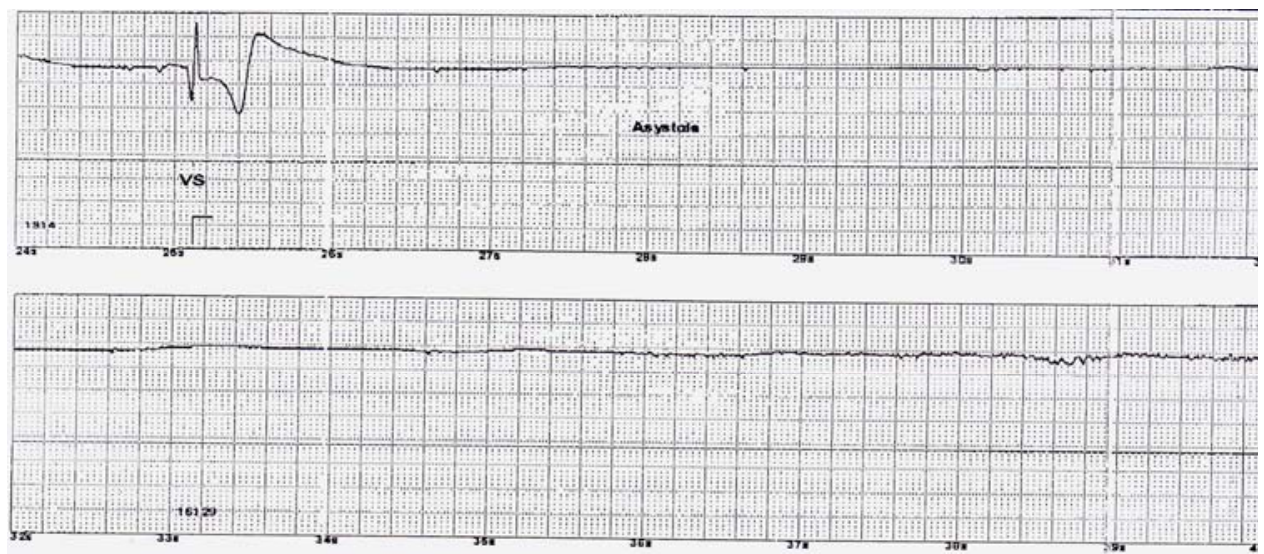

$-$

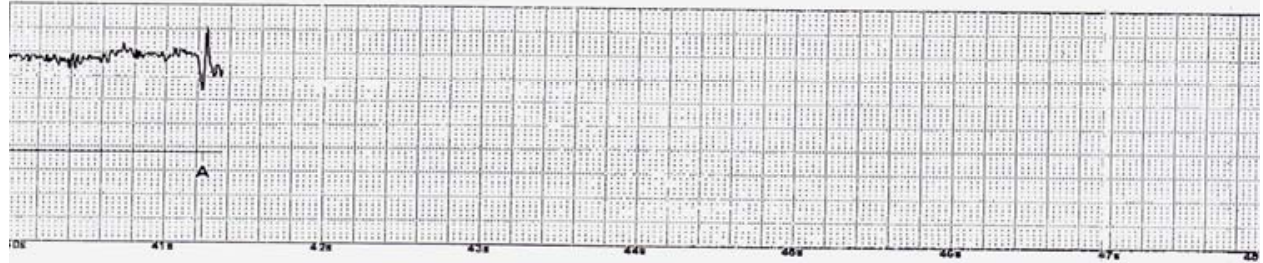

Figure 2: Asystole continues through out the tracing.
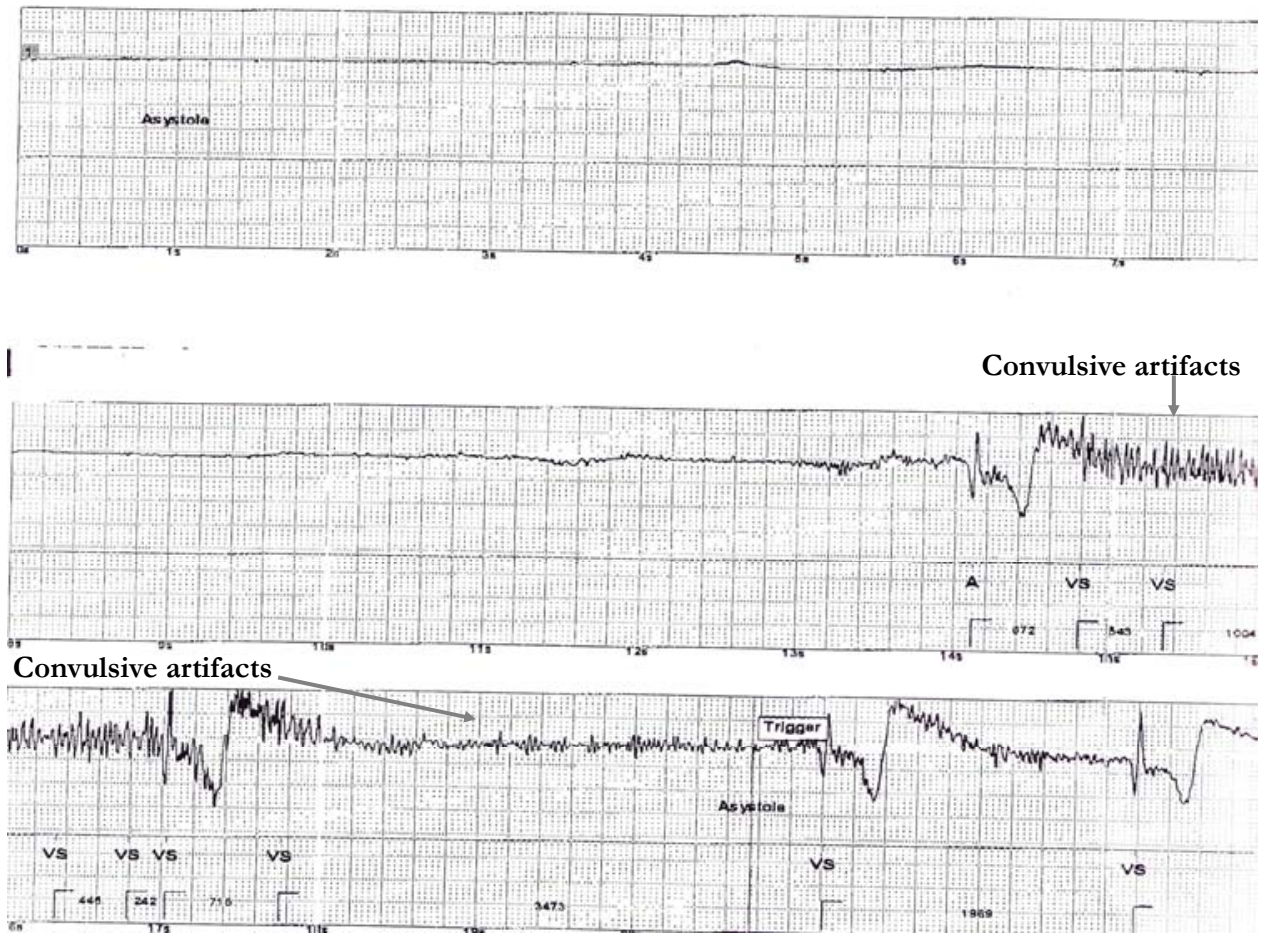

Figure 3: Prolonged asystole followed by a convulsive activity. 

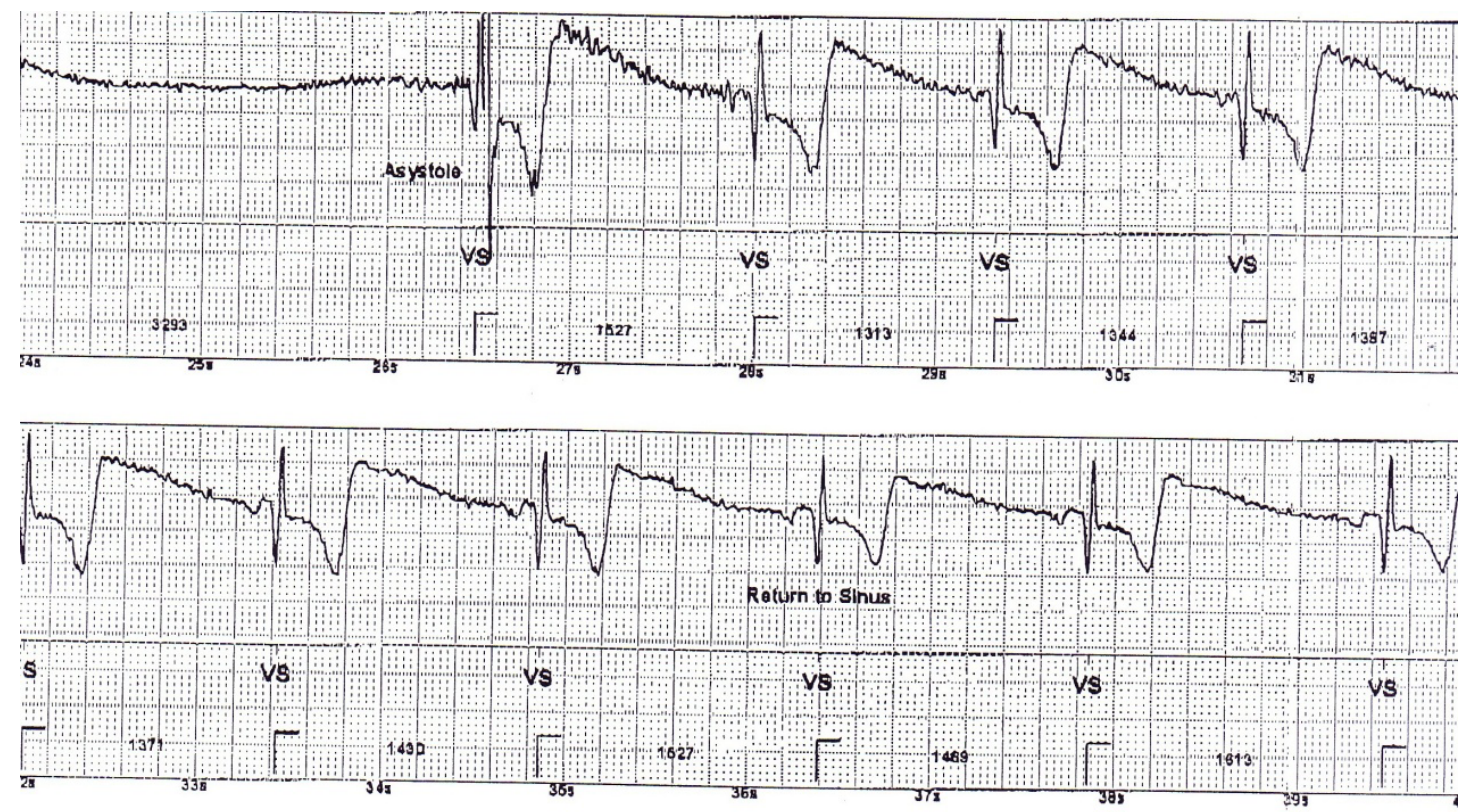

Figure 4: Tracing reveals return of patients' rhythm to Sinus. 